\title{
Os fatores sociodemográficos moderam a associação da prática de atividade física dos pais e amigos com o nível de atividade física dos adolescentes?
}

\author{
Sociodemographic factors moderate the association of the physical activity practice of \\ parents and friends with the level of physical activity of adolescents?
}

\section{AUTORES \\ Gerfeson Mendonça ${ }^{1,2}$ (iD) \\ Jacqueline de Oliveira Mendes ${ }^{1}$ (D) \\ Joana Marcela Sales de Lucena ${ }^{1}$ (D) \\ José Cazuza de Farias Júnior ${ }^{1,3,4}$ (iD \\ 1 Grupo de Estudos e Pesquisas em Epidemiologia \\ da Atividade Física, João Pessoal, Paraíba, Brasil. \\ 2 Centro Universitário Cesmac, Maceió, Alagoas, Brasil. \\ 3 Programa Associado de Pós-Graduação em Educação Física UPE/UFPB, João Pessoal, Paraíba, Brasil. \\ 4 Universidade Federal da Paraíba, João Pessoal, Paraíba, Brasil.}

\section{CONTATO}

Gerfeson Mendonça

gerfeson_edf@hotmail.com

Departamento de Educação Física, Universidade Federal da Paraíba. Campus I, Cidade Universitária, João Pessoa, Pernambuco,

Brasil.

CEP: 58059-900.

DOI

10.12820/rbafs.24e0092

\section{(cc) BY-NC-SA}

Este obra está licenciado com uma Licença Creative Commons Atribuicão-NãoComercialCompartilhaIgual 4.0 Internacional.

\begin{abstract}
RESUMO
O objetivo deste estudo foi analisar se os fatores sociodemográficos moderam a associação da prática de atividade física dos pais e dos amigos com o nível de atividade física dos adolescentes. Trata-se de um estudo epidemiológico transversal, realizado com 2.859 adolescentes (57,8\% sexo feminino) de 14 a 19 anos de idade do município de João Pessoa, Paraíba. O nível de atividade física dos adolescentes e a prática de atividade física dos pais e dos amigos foram mensurados por questionários. A prevalência de adolescentes fisicamente ativos foi de 50,2\% (IC95\%: 47,3-53,1), sendo mais elevada no sexo masculino (66,3\%; IC95\%: 63,7-69,0) comparada ao feminino (38,4\%; IC95\%: 36,1-40,8). Os resultados da regressão logística binária demostraram que há associação entre a prática de atividade física dos pais e dos amigos e o nível de atividade física dos adolescentes $(\mathrm{p}<0,001)$. No entanto, essa associação é moderada por fatores sociodemográficos (sexo e classe econômica). A magnitude da medida de associação entre a prática de atividade física dos amigos e a dos adolescentes foi mais elevada nos adolescentes do sexo masculino $(\mathrm{OR}=5,02 ; \mathrm{IC} 95 \%$ : 2,73-9,26 vs. feminino $\mathrm{OR}=2,21$; IC95\%: 1,55-3,14) e de classe econômica A/B (OR = 3,90; IC95\%: 2,39-6,39 vs. classe C/D/E: OR $=2,27$; IC95\%: 1,53-3,37). A prática de atividade física da mãe se associou de forma significativa e positiva com o nível de atividade física nos adolescentes de classe econômica $\mathrm{A} / \mathrm{B}(\mathrm{OR}=1,87$; IC95\%: 1,32-2,62). Conclui-se que os fatores sociodemográficos moderam a associação da prática de atividade física dos amigos e da mãe com o nível de atividade física dos adolescentes. A magnitude da associação entre essas variáveis variou com o sexo e a classe econômica dos adolescentes.
\end{abstract}

Palavras-chave: Atividade motora; Adolescência; Familiares; Colegas.

\section{ABSTRACT}

The objective of this study was to analyze if the sociodemographic factors moderate the association of physical activity practice of parents and friends, and the level of physical activity among adolescents. It was an cross sectional epidemiological study with 2,859 adolescents (57.8\% female) 14-19 years old of the city of Joano Pessoa, Paraiba. The level of physical activity among adolescents and the physical activity of parents and friends were measured by questionnaires. The prevalence of physically active adolescents was 50.2\% (95\% CI: 47.3-53.1), being higher in males (66.3\%; 95\%CI: 63.7-69.0) compared to female (38.4\%; 95\%CI: 36.140.8). The results of binary logistic regression analysis showed that there is an association between physical activity of parents and friends and the level of physical activity among adolescents $(p<0.001)$. However, this association is moderated by sociodemographic factors (sex and economic class). The magnitude of the measure of the association between the practice of physical activity of friends and adolescents was higher in male (OR $=5.02 ; 95 \% C I: 2.73-9.26$ vs. female $O R=2.21 ; 95 \% C I: 1.55-3.14)$ and $A / B$ economic class adolescents $(O R=3.90 ; 95 \% C I: 2.39-6.39$ vs. class $C / D / E: O R=2.27 ; 95 \% C I: 1.53-3.37)$. The physical activity of the mother was associated significantly and positively with the level of physical activity in adolescents of $A / B$ economic class (OR $=1.87 ; 95 \% C I: 1.32-2.62)$. We conclude that sociodemographic factors moderate the association of physical activity from friends and from the mother with the level of adolescents physical activity. The magnitude of the association between these variables varied with sex and economic class of adolescents.

Keywords: Motor activity; Adolescents; Relatives; Peers.

\section{Introdução}

A atividade física na adolescência tem sido alvo de diversas investigações ${ }^{1-5}$, sobretudo as que buscam entender qual o motivo de alguns adolescentes serem fisica- mente ativos e outros não $0^{3,5,6}$. A influência social está entre os fatores correlatos mais investigados e que tem se associado à atividade física dos adolescentes ${ }^{3,5-7}$. Essa influência pode acontecer pelo fornecimento de apoio 
social, normas e modelação do comportamento em relação à prática de atividade física ${ }^{7,8}$.

A modelação do comportamento para a prática de atividade física por parte dos pais e dos amigos representa uma das formas de influência social mais investigada $^{7,9,10}$. Tem sido caracterizada como uma aprendizagem observacional, na qual o comportamento de pessoas socialmente importantes para os adolescentes passa a ser adotado por estes como um modelo de comportamento ${ }^{9}$. Nesse sentido, a prática de atividade física dos pais e dos amigos pode representar um modelo para a prática dos adolescentes ${ }^{5,8,11}$.

Estudos que analisaram as relações entre a prática de atividade física dos pais e a atividade física dos filhos (adolescentes) demonstraram que pais fisicamente ativos são mais propensos a terem filhos igualmente ativos fisicamente ${ }^{5,11,12}$. O mesmo tem sido identificado em relação à prática de atividade física dos amigos e a dos adolescentes ${ }^{13,14}$, apesar do número de estudos ainda ser relativamente pequeno comparado aos dos pais $^{5,8,12}$. Entretanto, os resultados desses estudos são conflitantes ${ }^{8,11,12}$ para confirmarem as hipóteses de que essas associações variam conforme características sociodemográficas dos adolescentes como sexo, idade e classe econômica dos pais e dos amigos.

Os estudos têm demonstrado que os adolescentes do sexo feminino, os mais velhos e os que pertencem aos estratos socioeconômicos mais baixos são menos ativos fisicamente ${ }^{1,4}$. Desse modo, é possível que a influência da modelação dos hábitos de atividade física dos pais e dos amigos sobre a atividade física dos filhos (amigos) seja diferente entre esses subgrupos, indicando que os fatores sociodemográficos atuariam como moderadores dessa associação.

Em estudo de revisão sistemática, Seabra et al. ${ }^{15}$ identificaram que não é consensual o efeito moderador do sexo em relação a influência da prática de atividade física do pai e/ou da mãe sobre o nível de atividade física dos filhos e filhas, respectivamente. Em uma meta-análise, Yao \& Rhodes ${ }^{16}$ mostram que há resultados de estudos que apontam associações significativas entre a atividade física da mãe e das filhas e entre a do pai e a dos filhos, e outros resultados identificaram associações significativas entre a atividade física do pai e da mãe com os filhos de ambos os sexos. Esse mesmo cenário também tem sido observado em relação ao sexo dos adolescentes quanto à atividade física dos amigos ${ }^{15,16}$. Em relação ao possível efeito moderador da idade e da condição socioeconômica na associação entre a prática de atividade física dos pais e dos amigos e a dos adolescentes são necessários mais estudos ${ }^{15,16}$.

Identificar e compreender quais fatores sociodemográficos moderam as associações entre a prática de atividade física dos pais e dos amigos e a dos adolescentes ajudará a esclarecer em quais subgrupos (sexo, idade e classe econômica) a prática de atividade física desses pode exercer maior influencia sobre a atividade física dos adolescentes. Essas informações podem ajudar a orientar as intervenções sobre atividade física em relação ao envolvimento dos pais e dos amigos conforme as características sociodemográficas dos adolescentes. $\mathrm{O}$ objetivo deste estudo foi analisar se fatores sociodemográficos moderam a associação da prática de atividade física dos pais e dos amigos com o nível de atividade física dos adolescentes.

\section{Métodos}

Estudo epidemiológico transversal, de base escolar, baseado na análise dos dados de um estudo mais amplo intitulado "Nível de atividade física e fatores associados em adolescentes do ensino médio na cidade de João Pessoa, Paraíba: uma abordagem ecológica”, com adolescentes de ambos os sexos, de 14 a 19 anos de idade, de escolas do ensino médio das redes pública e privada no município de João Pessoa, Paraíba, realizada em 2009.

Para determinar o tamanho da amostra, os seguintes parâmetros foram adotados: prevalência estimada de 50\% (300 minutos ou mais por semana de atividade física); erro máximo aceitável de três pontos percentuais; intervalo de confiança de 95\%; efeito de desenho (deff) igual a dois e acréscimo de $30 \%$ para perdas e recusas, resultando em uma amostra estimada em 2.686 adolescentes.

A seleção da amostra foi por conglomerados em dois estágios (seleção sistemática de 30 escolas, distribuídas proporcionalmente por tipo [públicas e privadas], tamanho [número de alunos matriculados] e regiões do município [norte, sul, leste, oeste]; seguida da seleção aleatória de 135 turmas, distribuídas proporcionalmente por turno escolar [diurno e noturno] e série do ensino médio [1 $1^{\mathrm{a}}, 2^{\mathrm{a}}$ e $3^{\mathrm{a}}$ séries]).

A coleta de dados ocorreu no período de maio a setembro de 2009 e foi realizada por uma equipe de seis estudantes de graduação em Educação Física, treinados e submetidos a um estudo piloto sob as mesmas condições do estudo principal. Todas as informações foram coletadas por meio de questionário, previamente testado, preenchido pelos próprios adolescentes, em sala de aula, seguindo instruções prévias fornecidas pela equi- 
pe de coleta, durante todo o processo para esclarecer dúvidas e orientar a forma correta de preenchimento. Para reduzir as perdas, o questionário foi aplicado de terça a quinta-feira, no segundo horário de aula.

As variáveis sociodemográficas analisadas neste estudo foram: sexo (masculino e feminino); idade em anos completos (categorizada em "14-15" e "16-19 anos de idade"); classe econômica (metodologia da Associação Brasileira das Empresas de Pesquisa - ABEP) ${ }^{17}$, que leva em consideração a presença de bens materiais e de empregado mensalista na residência, e a escolaridade dos pais. A partir desses indicadores, as pessoas foram agrupadas nas classes econômicas A1, A2, B1, B2, C1, $\mathrm{C} 2$, D e E. Para fins de análise, elas foram reagrupadas em classes $\mathrm{A} / \mathrm{B}$ (alta) e $\mathrm{C} / \mathrm{D} / \mathrm{E}$ (média-baixa).

$\mathrm{O}$ estado nutricional foi mensurado pelo índice de massa corporal $(\mathrm{IMC}=$ massa corporal $[\mathrm{kg}] /$ estatura $[\mathrm{m}]^{2}$ ), baseado nas medidas autorreferidas de massa corporal $(\mathrm{kg})$ e estatura $(\mathrm{cm})^{18}$. Os adolescentes foram categorizados em "sem excesso de peso corporal" (baixo peso + peso normal) e "com excesso de peso corporal" (sobrepeso + obesidade), conforme os pontos de corte sugeridos por Cole \& Lobstein ${ }^{19}$.

A prática de atividade física do pai, da mãe e dos amigos foi mensurada pela seguinte questão: "Durante uma semana típica ou normal, em quantos dias... (sua mãe / seu pai / seus amigos), pratica(m) atividades físicas como, por exemplo, caminhar, correr, andar de bicicleta, ir à academia de ginástica, fazer esportes ou musculação?”. As opções de respostas variavam de "nenhum" a "cinco ou mais dias por semana" e foram categorizadas em "nenhum dia", "1 a 2 dias" ou " 3 ou mais dias”. Essas questões tiveram os seguintes níveis de reprodutibilidade teste-reteste (coeficiente de correlação intraclasse - CCI): prática de atividade física do pai - CCI = 0,92 (IC95\%: 0,90-0,94), da mãe - CCI = 0,90 (IC95\%: 0,87 - 0,92) e dos amigos $-\mathrm{CCI}=0,82$ (IC95\%: 0,76-0,86).

A medida de atividade física dos adolescentes foi efetuada por questionário que apresentou altos níveis de reprodutibilidade: $\mathrm{CCI}=0,88$; IC95\%: 0,84-0,91) e validade (Spearman's rho $=0,61 ; \mathrm{p}<0,01)^{20}$. Os adolescentes informaram a frequência (dias/semana) e a duração (minutos/dia) das atividades físicas de intensidade moderada a vigorosa e do deslocamento ativo, praticados por pelo menos 10 minutos, na semana que antecedeu a coleta de dados, tomando como base uma lista com 24 atividades, com possibilidade dos adolescentes acrescentarem até duas atividades físicas. $\mathrm{O}$ nível de atividade física foi determinado pelo somatório dos produtos do tempo despendido nessas atividades e pelas suas respectivas frequências de prática. Os adolescentes com prática de atividade física igual ou superior a 300 minutos por semana foram considerados como "fisicamente ativo", e os demais como "fisicamente inativo" 5,21 .

Os dados foram digitados no programa EpiData 3.1, seguindo um processo de dupla digitação, com checagem automática de consistência e amplitude dos valores de cada variável. A ferramenta "validar dupla digitação" desse programa foi utilizada para identificar possíveis erros de digitação. Todos os erros foram identificados e corrigidos com base nos valores registrados nos respectivos questionários.

Para a análise descritiva dos dados foi observada a distribuição por frequência, média, desvio padrão (DP) e intervalo de confiança de 95\%. O teste de Qui-quadrado para tendência linear foi utilizado para comparar a proporção de adolescentes classificados como fisicamente ativos de acordo com a prática de atividade física dos pais e dos amigos.

A regressão logística binária foi utilizada para verificar a associação entre a prática de atividade física da mãe, do pai e dos amigos (variáveis independentes) e o nível de atividade física dos adolescentes (variável dependente). As variáveis independentes foram introduzidas no mesmo nível de análise e mantidas no modelo múltiplo (análise ajustada). Para avaliar se os fatores sociodemográficos (sexo, idade e classe econômica) moderavam essas associações, foram introduzidos termos de interação (multiplicação da possível variável moderadora com a variável independente - por exemplo: sexo*atividade física da mãe) no modelo. Termo de interação com valor $\mathrm{p}<0,05$ foi considerado como significativo, indicando a presença de moderação da variável sociodemográfica em análise. Nestes casos, foram realizadas análises estratificadas por cada variável sociodemográfica classificada como moderadora. Isso permitiu avaliar o sentido e a magnitude da medida de associação entre o nível de atividade física dos adolescentes e a prática de atividade física dos pais e dos amigos nos estratos dessas variáveis. As análises estatísticas foram realizadas no Stata 11.0. O nível de significância adotado foi de $\mathrm{p}<0,05$.

O estudo foi aprovado pelo Comitê de Ética em Pesquisa com Seres Humanos do Centro de Ciências da Saúde da Universidade Federal da Paraíba - Protocolo: 0062/2009. Todos os adolescentes menores de 18 anos de idade que participaram do estudo receberam 
autorização dos pais ou responsáveis e aqueles com mais de 18 anos de idade assinaram o termo de consentimento livre e esclarecido (TCLE). Os adolescentes que não retornaram o TCLE foram considerados perdas amostrais. Os critérios de exclusão adotados foram: adolescentes fora da faixa etária estabelecida (menores de 14 anos e maiores de 19 anos); ter alguma deficiência que dificultasse ou limitasse a prática de atividade física e/ou os impedisse de preencher o questionário.

\section{Resultados}

A amostra foi composta por 2.859 adolescentes de 14 a 19 anos de idade (perdas e recusas totalizaram 17,8\% dos casos), com média de idade de 16 anos ( $\mathrm{DP}=1,2)$. Cálculos realizados a posteriori demonstraram que essa amostra tinha poder de $80 \%$ para detectar como significativas razões de odds iguais ou superiores a 1,40. A maioria dos adolescentes era do sexo feminino $(57,8 \%)$, tinha entre 16 e 19 anos de idade $(60,7 \%)$ e pertencia as classes econômicas média-baixa $(54,1 \%)$ - Tabela 1 . A proporção de adolescentes fisicamente ativos foi igual a 50,2\% (IC95\%: 47,3-53,1), sendo estatisticamente mais elevada no sexo masculino (66,3\%; IC95\%: 63,7-69,0) comparado ao feminino (38,4\%; IC95\%: 36,1-40,8). A maioria dos pais (50,6\%; IC95\%: 48,5-52,2) e amigos (88,1\%; IC95\%: 87,0-89,4) praticava atividades físicas em um ou mais dias por semana, e 49,4\% (IC95\%: 47,6-51,2) das mães apresentaram essa frequência de prática - Dados não apresentados em tabela.

$\mathrm{Na}$ análise multivariável, observou-se que a prática de atividade física de ambos os pais e dos amigos se associou de forma positiva e significativa com o nível de atividade física dos adolescentes, indicando que quanto maior a prática de atividade física desses grupos, maiores as chances dos adolescentes serem fisicamente ativos. A magnitude da medida de associação foi significativamente maior (sem intercessão dos valores de IC95\%) para a frequência de prática de atividade física dos amigos de 3 ou mais dias por semana $(\mathrm{OR}=3,01$; IC95\%: 2,23-4,06) em relação à prática da mãe (OR = 1,71; IC95\%: $1,40-2,09)$ e do pai (OR = 1,36; IC95\%: 1,33-2,00) - Tabela 2.

Foram identificadas interações significativas entre a prática de atividade física dos amigos e as variáveis sexo $(p<0,001)$ e classe econômica $(p=0,039)$, e entre a prática das mães e a classe econômica da família $(\mathrm{p}=$ $0,035)$. Não houve interação significativa entre a variável idade e a prática de atividade física dos pais e dos amigos $(p>0,05)-$ Tabela 2 .
Tabela 1 - Características da amostra de adolescentes do município João Pessoa, Paraíba, 2009 ( $\mathrm{n}=2.859$ )

\begin{tabular}{|c|c|c|}
\hline Variável & $\mathrm{n}^{*}$ & $\%$ \\
\hline \multicolumn{3}{|l|}{ Sexo } \\
\hline Masculino & 1.206 & 42,2 \\
\hline Feminino & 1.653 & 57,8 \\
\hline \multicolumn{3}{|l|}{ Idade } \\
\hline $14-15$ anos & 1.124 & 39,3 \\
\hline $16-19$ anos & 1.735 & 60,7 \\
\hline \multicolumn{3}{|l|}{ Cor da pele } \\
\hline Branco & 929 & 32,6 \\
\hline Não branco & 1.915 & 67,4 \\
\hline \multicolumn{3}{|l|}{ Trabalho } \\
\hline Trabalha & 364 & 13,1 \\
\hline Não trabalha & 2.424 & 86,9 \\
\hline \multicolumn{3}{|l|}{ Classe econômica } \\
\hline $\mathrm{A} / \mathrm{B}$ (alta) & 1.158 & 45,9 \\
\hline C/D/E (média-baixa) & 1.366 & 54,1 \\
\hline \multicolumn{3}{|l|}{ Escolaridade do pai } \\
\hline Fundamental & 1.384 & 49,9 \\
\hline Médio completo & 893 & 32,2 \\
\hline Superior completo & 495 & 17,9 \\
\hline \multicolumn{3}{|l|}{ Escolaridade da mãe } \\
\hline Fundamental & 1.383 & 48,8 \\
\hline Médio completo & 901 & 31,8 \\
\hline Superior completo & 549 & 19,4 \\
\hline \multicolumn{3}{|c|}{ Prática de atividade física da mãe } \\
\hline $0 \mathrm{dia} / \mathrm{semana}$ & 1.435 & 50,6 \\
\hline 1-2 dias/semana & 611 & 21,6 \\
\hline 3 ou mais dias/semana & 786 & 27,8 \\
\hline \multicolumn{3}{|c|}{ Prática de atividade física do pai } \\
\hline 0 dia/semana & 1.374 & 49,6 \\
\hline 1-2 dias/semana & 659 & 23,8 \\
\hline 3 ou mais dias/semana & 734 & 26,6 \\
\hline \multicolumn{3}{|c|}{ Prática de atividade física dos amigos } \\
\hline $0 \mathrm{dia} / \mathrm{semana}$ & 333 & 11,8 \\
\hline 1-2 dias/semana & 965 & 34,3 \\
\hline 3 ou mais dias/semana & 1.518 & 53,9 \\
\hline \multicolumn{3}{|c|}{ Atividade física dos adolescentes } \\
\hline Fisicamente inativo & 1.423 & 49,8 \\
\hline Fisicamente ativo & 1.436 & 50,2 \\
\hline
\end{tabular}

* Diferenças no $n^{\circ}$ de 2.859 adolescentes são referentes ao ausência de resultados válidos (missing) na variável.

$\mathrm{Na}$ Figura 1 foram apresentados os resultados das análises de prática de atividade física dos amigos e das mães e o nível de atividade física dos adolescentes estra- 
Tabela 2 - Análise de regressão logística binária bruta e ajustada para associação entre prática de atividade física dos pais e dos amigos e o nível de atividade física dos adolescentes de João Pessoa, Paraíba, 2009 ( $\mathrm{n}=2.859$ )

\begin{tabular}{|c|c|c|c|c|c|c|c|c|c|c|}
\hline \multirow{2}{*}{ Variáveis } & \multicolumn{4}{|c|}{ Prevalência } & \multicolumn{3}{|c|}{ Análise bruta } & \multicolumn{3}{|c|}{ Análise ajustada* } \\
\hline & $\mathrm{n}$ & $\%$ & IC $95 \%$ & $\mathrm{p}$ & OR & IC95\% & $\mathrm{p}$ & OR & IC95\% & $\mathrm{p}$ \\
\hline Prática de atividade física da mãe & & & & $<0,001$ & & & $<0,001$ & & & $<0,001$ \\
\hline $0 \mathrm{dia} / \mathrm{semana}$ & 649 & 45,0 & $42,5-47,6$ & & 1,00 & & & 1,00 & & \\
\hline 1-2 dias/semana & 343 & 55,7 & $51,7-59,6$ & & 1,53 & $1,27-1,85$ & & 1,41 & $1,13-1,75$ & \\
\hline 3 ou mais dias/semana & 444 & 56,2 & $52,7-59,6$ & & 1,57 & $1,31-1,86$ & & 1,71 & $1,40-2,09$ & \\
\hline Prática de atividade física do pai & & & & $<0,001$ & & & $<0,001$ & & & $<0,001$ \\
\hline $0 \mathrm{dia} / \mathrm{semana}$ & 632 & 45,8 & $43,1-48,4$ & & 1,00 & & & 1,00 & & \\
\hline 1-2 dias/semana & 369 & 55,7 & $51,9-59,5$ & & 1,49 & $1,24-1,80$ & & 1,35 & $1,09-1,67$ & \\
\hline 3 ou mais dias/semana & 408 & 55,3 & $51,7-58,8$ & & 1,47 & $1,22-1,75$ & & 1,36 & $1,33-2,00$ & \\
\hline Prática de atividade física dos amigos & & & & $<0,001$ & & & $<0,001$ & & & $<0,001$ \\
\hline $0 \mathrm{dia} /$ semana & 95 & 28,4 & $23,8-33,5$ & & 1,00 & & & 1,00 & & \\
\hline 1-2 dias/semana & 403 & 41,6 & $38,5-44,7$ & & 1,79 & $1,37-2,35$ & & 1,49 & $1,09-2,03$ & \\
\hline 3 ou mais dias/semana & 934 & 61,1 & $58,7-63,5$ & & 3,96 & $3,05-5,13$ & & 3,01 & $2,23-4,06$ & \\
\hline \multicolumn{11}{|l|}{ Interações } \\
\hline \multicolumn{4}{|c|}{ Sexo $x$ Prática de atividade física dos amigos** } & & & & & 1,67 & $1,25-2,23$ & $<0,001$ \\
\hline \multicolumn{4}{|l|}{ Sexo x Prática de atividade física do pai } & & & & & 1,24 & $0,99-1,55$ & 0,051 \\
\hline \multicolumn{4}{|l|}{ Sexo x Prática de atividade física da mãe } & & & & & 0,98 & $0,79-1,21$ & 0,851 \\
\hline \multicolumn{4}{|c|}{ Idade $\mathrm{x}$ Prática de atividade física dos amigos } & & & & & 1,05 & $0,80-1,38$ & 0,676 \\
\hline \multicolumn{4}{|l|}{ Idade $\mathrm{x}$ Prática de atividade física do pai } & & & & & 0,94 & $0,76-1,17$ & 0,606 \\
\hline \multicolumn{4}{|c|}{ Idade $x$ Prática de atividade física da mãe } & & & & & 1,07 & $0,87-1,32$ & 0,493 \\
\hline \multicolumn{4}{|c|}{ Classe econômica $\mathrm{x}$ Prática de atividade física dos amigos** } & & & & & 1,32 & $1,01-1,72$ & 0,039 \\
\hline \multicolumn{4}{|c|}{ Classe econômica x Prática de atividade física do pai } & & & & & 1,17 & $0,95-1,44$ & 0,138 \\
\hline \multicolumn{4}{|c|}{ Classe econômica x Prática de atividade física da mãe $\mathrm{e}^{* *}$} & & & & & 1,24 & $1,01-1,53$ & 0,035 \\
\hline
\end{tabular}

*Ajustada por sexo, idade, classe econômica e índice de massa corporal; IC95\% = Intervalo de confiança de 95\%.*Diferença significativa (p < $0,05)$ na análise de interação.

tificadas por sexo e classe econômica. Os resultados dessas análises demonstraram que a magnitude da medida de associação entre a prática de atividade física dos amigos e o nível de atividade física dos adolescentes do sexo masculino foi mais que o dobro $(\mathrm{OR}=5,02$; IC95\%: 2,73-9,26) quando comparada a do sexo feminino (OR = 2,21; IC95\%: 1,55-3,14) - Figura 1A. Já a magnitude da medida de associação entre a prática de atividade física dos amigos e o nível de atividade física dos adolescentes de classe econômica alta foi maior $(\mathrm{OR}=$ 3,90; IC95\%: 2,39-6,39) do que a identificada na classe econômica média-baixa (OR = 2,27; IC95\%: 1,53-3,37) - Figura 1B. Nos adolescentes de classe econômica alta foi identificado aumento de até $80 \%$ na chance de serem fisicamente ativos quando a mãe também era fisicamen- te ativa 3 ou mais dias por semana (Figura 1C).

Diante da tendência linear de aumento na prevalência de adolescentes fisicamente ativos, conforme a prática de atividade física do pai, da mãe e dos amigos (Tabela 2), verificou-se maior prevalência nos adolescentes do sexo masculino para a prática dos amigos (1-2 dias/sem. $=52,6 \%$; IC95\%: 47,3-57,9; 3 ou mais dias/sem. = 75,3\%; IC95\%: 72,2-78,2) em relação ao sexo feminino (1-2 dias/sem. = 35,4\%; IC95\%: 31,839,3; 3 ou mais dias/sem. = 46,2\%; IC95\%: 42,6-49,8), e nos de classe econômica $\mathrm{A} / \mathrm{B}$ quando a prática dos amigos foi frequente em 3 ou mais dias por semana (62,9\%; IC95\%: 57,4-68,0) comparado aos de classe C/D/E (52,3\%; IC95\%: 47,3-57,3) - resultados não disponíveis em tabela. 
a)

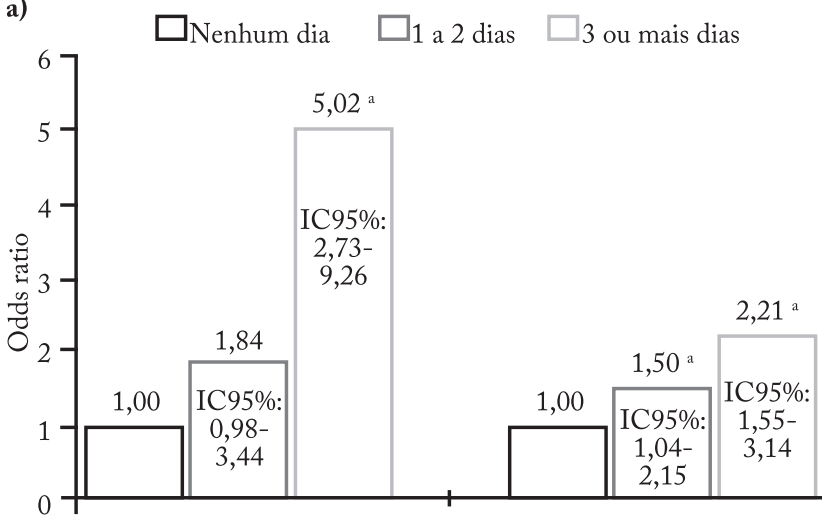

b)

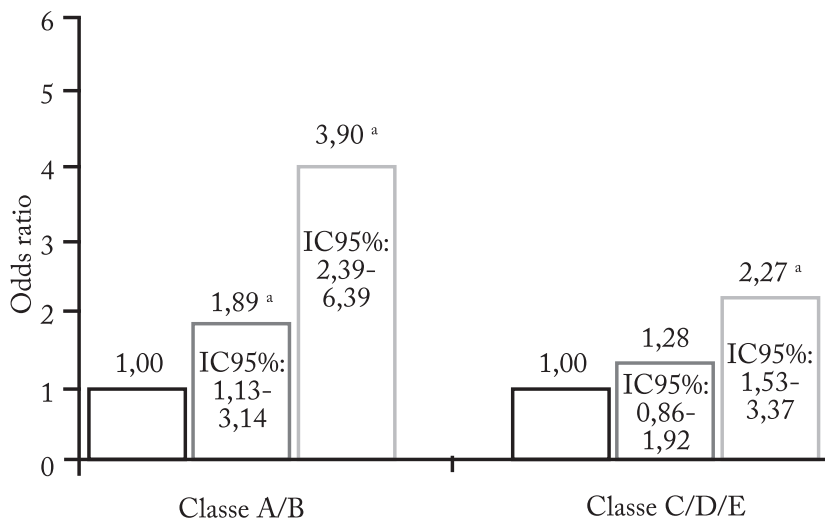

c)

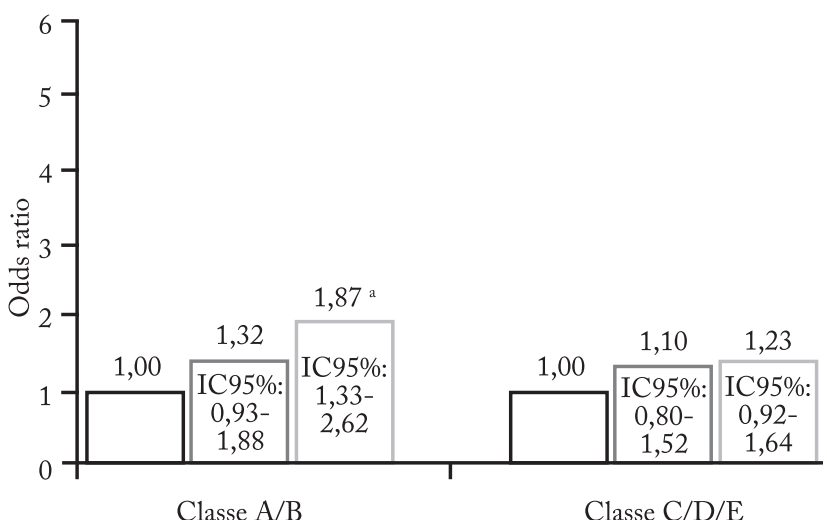

Figura 1 - Análise de regressão logística binária ajustada* para associação entre prática de atividade física dos pais e dos amigos e o nível de atividade física dos adolescentes de João Pessoa, Paraíba, 2009 ( $\mathrm{n}=2.859$ )

A) Odds ratio da associação entre prática dos amigos e nível de atividade física dos adolescentes estratificado por sexo; B) Odds ratio da associação entre prática dos amigos e nível de atividade física dos adolescentes estratificado por classe econômica; C) Odds ratio da associação entre prática das mães e nível de atividade física dos adolescentes estratificado por classe econômica. IC95\% = Intervalo de confiança de 95\%. *Ajustada por sexo, idade, classe econômica e índice de massa corporal; $\mathbf{a}=$ associação significativa (valor $\mathrm{p}<0,05$ )

\section{Discussão}

O principal objetivo deste estudo foi analisar se os fatores sociodemográficos moderam a associação da prática de atividade física dos pais e dos amigos com o nível de atividade física dos adolescentes. De forma geral, foi identificado que maior prática de atividade física dos pais e dos amigos estava associada com maiores níveis de atividade física dos adolescentes. No entanto, a magnitude das medidas de associação entre a prática dos amigos e o nível de atividade física dos adolescentes foi maior para os do sexo masculino e de classe econômica alta em relação a seus pares. Também foi identificado que a prática da mãe se associou com o nível de atividade física nos adolescentes de classe econômica alta. Esses resultados, em conjunto, indicam que os fatores sociodemográficos moderam a associação entre essas variáveis.

No presente estudo, adolescentes que percebiam seus pais e, sobretudo, os seus amigos participando de atividades físicas com maior frequência, apresentaram maiores chances de serem fisicamente mais ativos. Esses resultados são semelhantes aos de outros estudos $^{11-13}$. A modelagem do comportamento está entre as principais formas de influência social exercida pelos principais grupos sociais de convívio dos adolescentes, sendo frequentemente citada como um importante fator que influência a atividade física desse grupo ${ }^{5,13}$. A modelação por meio da observação direta e o convívio dos adolescentes com os pais e os amigos são aspectos que podem determinar a adesão de comportamentos semelhantes ${ }^{9}$, dentre eles a prática de atividade física.

Como observado em estudos com adolescentes de diferentes países ${ }^{11,13}$, no presente estudo, verificou-se uma maior magnitude de associação entre o nível de atividade física dos adolescentes e a prática de atividade física dos amigos do que a prática dos pais (pai e mãe). É possível que o efeito da modelação do comportamento para a prática de atividade física seja maior nos amigos, uma vez que com o passar dos anos, os adolescentes tendem a permanecer mais tempo na companhia dos amigos do que dos pais ${ }^{11}$. Sendo assim, passariam a ser mais influenciados pelos hábitos e normas partilhados com os amigos ${ }^{13,22}$. Outra explicação pode estar atrelada a possibilidade de que os adolescentes recordariam com maior precisão da participação dos amigos em atividades físicas por serem essas práticas realizadas, em sua maioria, de forma conjunta. Normalmente, os adolescentes partilham das mesmas atividades que seus amigos, frequentam ambientes de 
prática semelhantes, dividem os gostos pelas mesmas atividades e adotam comportamentos parecidos de prática para pertencer ao seu grupo social ${ }^{13}$. Em estudo de revisão sistemática, Sawka et al. ${ }^{22}$ identificaram que os adolescentes podem adotar um comportamento em decorrência da influência social exercida pelos amigos ou por meio da seleção de amigos que apresentassem alguns interesses em comum. No que se refere a atividade física, a escolha das amizades estaria relacionada a aproximação dos adolescentes igualmente ativos fisicamente $^{22}$. Além disso, é importante reforçar a grande influência que o apoio social de amigos tem sobre a prática dos adolescentes, tendo em vista o fato de que na adolescência há uma necessidade maior de se incluir em grupos de amigos ${ }^{14,23}$.

Apesar de a prática de atividade física dos amigos ter se mostrado associada a maiores níveis de atividade física em adolescentes de ambos os sexos, a magnitude da associação foi significativamente maior no sexo masculino comparado ao feminino. Esse achado indica o efeito moderador dessa variável para essa associação, aspecto que também foi observado em outros estudos com adolescentes ${ }^{14,15,23}$. A maior participação dos adolescentes do sexo masculino em atividades físicas coleti$\operatorname{vas}^{24,25}$, o fato de receberem mais apoio social para a prática de atividade física dos seus ciclos de amizades ${ }^{14,23} \mathrm{e}$ estarem mais envolvidos em atividades realizadas ao ar livre ${ }^{25}$, são aspectos que aumentam a possibilidade de novas amizades e consecutivamente um maior potencial de modelação de suas práticas de atividade física.

É importante destacar também que os adolescentes do sexo masculino praticam e valorizam mais a participação em esportes, por exemplo, como futebol e voleiboll $^{25}$. Sendo assim, é possível que eles se espelhem nos seus ídolos dessas modalidades. Por outro lado, as adolescentes apresentam menor nível de prática de atividade física, principalmente em esportes ${ }^{1,2,4}$. Outro aspecto que precisa ser considerado é o fato de que as adolescentes são estimuladas desde cedo para se envolverem em atividades domésticas e seguirem "regras sociais" ou "estigmas" que as induzem a permanecer mais tempo dentro de casa ${ }^{24}$. Isso pode contribuir para diminuir as oportunidades de maior envolvimento com outros adolescentes a quem pudessem se espelhar para maiores práticas.

O presente estudo identificou que a classe econômica moderou a associação entre a prática de atividade física dos amigos e o nível de atividade física dos adolescentes. Apesar dessas variáveis estarem positivamente associadas nos adolescentes de classes econômicas alta e média-baixa, a magnitude de associação foi maior nos de classe econômica alta. Adolescentes de condição econômica alta apresentam maiores oportunidades de encontrarem amigos e realizarem suas atividades físicas nos períodos de lazer, principalmente em atividades estruturadas e/ou coletivas, diferentemente daqueles de menor condição socioeconômica ${ }^{26}$. Esse argumento pode colaborar para explicar os resultados do presente estudo, tendo em vista que para que essas práticas aconteçam frequentemente há a necessidade de locais específicos (por exemplo: quadras, ginásios esportivos, academias e clubes recreativos) ${ }^{8,11,12}$. Esses locais seriam mais acessíveis para as pessoas de classe socioeconômica mais alta, pois em grande parte dependem de recursos financeiros, materiais esportivos e transporte até os locais $^{26}$. Dessa forma, possibilitariam maiores vínculos de amizade, consequentemente, os adolescentes poderiam se espelhar nas práticas de atividade física desses amigos.

Outro achado importante deste estudo foi identificar a associação entre maior prática de atividade física das mães e os maiores níveis de atividade física nos adolescentes de classe econômica alta, caracterizando o efeito moderador dessa variável. Esse resultado pode estar relacionado ao fato de que as mães de melhor condição socioeconômica, geralmente, residem em bairros com melhor infraestrutura para a prática de atividades físicas (presença de parques, praças, pista de corrida/caminhada, ciclovias ou ciclofaixas) $)^{5}$, possuem maior grau de conhecimento sobre a adoção de comportamentos positivos à saúde, tendo assim maiores chances de se tornarem mais fisicamente ativas. Sendo assim, elas passariam a serem exemplos ou referências para seus filhos (adolescentes) em termos de modelo para a prática de atividade física ${ }^{5,24}$.

É possível que entre as mães de condição econômica alta a prática de atividade física seja mais valorizada, e estas por sua vez podem transmitir maior apoio social para as práticas de seus filhos, por meio de palavras de encorajamento, fornecimento de transporte, praticar em conjunto ou assistir a prática deles em atividades físicas ${ }^{14}$. Já as mães de menor condição socioeconômica são frequentemente menos ativas fisicamente ${ }^{1,4}$, apresentam menor nível de conhecimento sobre a importância da atividade física para a saúde, fornecem menos apoio social para a atividade física dos filhos ${ }^{14}$, servindo possivelmente como modelos negativos para a prática deles. Mães de menor nível socioeconômico exigem maior envolvimento dos seus filhos em compromissos sociais como trabalhar, cuidar da casa, realizar cursos e 
estágios para capacitação ${ }^{12}$, resultando em menor nível de prática de atividade física por partes destes.

O presente estudo apresenta como pontos fortes: a utilização de uma amostra representativa de adolescentes do ensino médio de escolas públicas e privadas; o instrumento utilizado na coleta de dados foi previamente validado e a coleta de dados foi realizada por equipe treinada. Entretanto, o presente estudo apresenta algumas limitações: trata-se de um estudo transversal, não sendo possível estabelecer uma relação causal entre a prática de atividade física dos pais e dos amigos dos adolescentes; a medida da atividade física dos pais e dos amigos foi baseada na percepção do adolescente e não considerou o tempo de prática por dia. Esse aspecto pode ter superestimado a prática de atividade física dos pais e dos amigos quando essas práticas foram relatadas pelos adolescentes mais ativos e, consequentemente, superestimou as magnitudes das medidas de associação entre as variáveis em estudo.

Conclui-se que a associação entre a prática de atividade física dos amigos e das mães e o nível de atividade física dos adolescentes variou conforme o sexo e a classe econômica da família, demonstrando que os fatores sociodemográficos moderam a associação entre essas variáveis. Nos adolescentes do sexo masculino e de classe econômica alta foram identificadas maiores magnitudes nas medidas de associação entre a prática de atividade física dos amigos e a dos adolescentes. A prática de atividade física da mãe se associou apenas ao nível de atividade física dos adolescentes de classe econômica alta.

No desenvolvimento de intervenções para promoção da atividade física em adolescentes devem investir em ações que possibilitem um maior envolvimento dos pais e dos amigos com a prática de atividade física para que estes possam influenciar por meio da modelação do comportamento a prática de atividade física dos adolescentes. No entanto, as ações propostas devem considerar as caraterísticas sociodemográficas dos adolescentes, tendo em vista que a prática de atividade física dos amigos e das mães parecem exercer maior influência no nível de atividade física dos adolescentes do sexo masculino e os de classe econômica mais alta.

\section{Conflito de interesses}

Os autores declaram não haver conflito de interesses.

\section{Contribuições dos autores}

Mendonça G e Mendes JO, participaram da concepção do artigo, análise dos dados, revisão de literatura e redação do manuscrito. Lucena JMS, colaborou na revisão de literatura e redação do manuscrito. Farias Júnior JC, participou de todas as etapas do estudo, incluindo concepção do artigo, coleta e análise dos dados, redação e revisão crítica do manuscrito.

\section{Agradecimentos}

À Coordenação de Aperfeiçoamento de Pessoal de Nível Superior (CAPES) pela bolsa de estudos e pesquisa. À Secretaria de Educação da Paraíba, aos gestores, professores e alunos das escolas que participaram do estudo. Aos estagiários do Curso de Educação Física que fizeram parte da equipe de coleta de dados. Ao Programa de Iniciação Científica e Tecnológica - PIBIC da Universidade Federal da Paraíba.

\section{Referências}

1. Townsend N, Wickramasinghe K, Williams J, Bhatnagar P, Rayner M. Physical Activity Statistics 2015. London, UK: British Heart Foundation; 2015.

2. Mendonça G, Cheng LA, Farias Júnior JC. Padrões de prática de atividade física em adolescentes de um município da região Nordeste do Brasil. Ciênc. Saúde Coletiva. 2018;23(7):2443-51.

3. O'Donoghue G, Kennedy A, Puggina A, Aleksovska K, Buck C, Burns C, et al. Socio-economic determinants of physical activity across the life course: A "DEterminants of Dlet and Physical ACtivity” (DEDIPAC) umbrella literature review. PLoS ONE. 2018;13(1):e0190737.

4. Hallal P, Andersen LB, Bull FC, Guthold R, Haskell W, Ekelund U. Global physical activity levels: surveillance progress, pitfalls, and prospects. Lancet. 2012;380(9838):247-57.

5. De la Torre-Cruz MJ, López-Serrano S, Ruiz-Ariza A, Martínez-López EJ. Perceived parental support toward physical activity positively predicts physical self-concept in young adolescents. Educ Psychol. 2019;1(1):941-59.

6. Bauman AE, Reis RS, Sallis JF,Wells JC,Loos RJ, Martin BW. Correlates of physical activity: why are some people physically active and others not? Lancet. 2012;380(9838):258-71.

7. Chung SJ, Ersig AL, McCarthy AM. The Influence of Peers on Diet and Exercise Among Adolescents: A Systematic Review. J Pediatr Nurs. 2017;36(1):44-56.

8. Benes D, Dowling J, Crawford S, Hayman LL. Social and Environmental Influences on Physical Activity Levels in Latina Adolescents. Public Health Nurs. 2017;34(2):101-11.

9. Bandura A. Social cognitive theory: An agentic perspective. Annu Rev Psychol. 2001;52(1):1-26.

10. Bandura A. Self-efficacy: Toward a unifying theory of behavioral change. New York, NY, US: Psychology Press; 1999.

11. Anderson Steeves ET, Johnson KA, Pollard SL, Jones-Smith J, Pollack K, Lindstrom Johnson S, et al. Social influences on eating and physical activity behaviours of urban, minority youths. Public Health Nutr. 2016;19(18):3406-16.

12. Garcia JM, Sirard JR, Larsen R, Bruening M, Wall M, Neumark-Sztainer D. Social and Psychological Factors Associated With Adolescent Physical Activity. J Phys Act Health. 2016;13(9):957-63.

13. Fitzgerald A, Fitzgerald N, Aherne C. Do peers matter? A review of peer and/or friends' influence on physical activity among American adolescents. J Adolesc. 2012;35(4):941-58. 
14. Mendonça G, Cheng LA, Melo EN, Farias Junior JC. Physical activity and social support in adolescents: a systematic review. Health Educ Res. 2014;30(5):822-39.

15. Seabra AF, Mendonça DM, Thomis MA, Anjos LA, Maia JA. Biological and socio-cultural determinants of physical activity in adolescents. Cad Saude Publica. 2008;24(4):72136.

16. Yao CA, Rhodes RE. Parental correlates in child and adolescent physical activity: a meta-analysis. Int J Behav Nutr Phys Act. 2015;12(1):10.

17. ABEP - Brazilian Association of Research Companies. Brazil Economic Classification Criterion; 2009 [citado em 2009 Nov 26]. Disponível em: http://www.abep.org/new/ codigosCondutas.aspx.

18. Farias Júnior JC. Validity of self-reported weight and height for adolescent nutritional status diagnosis. Rev Bras Saúde Matern Infant. 2007;7(2):167-74.

19. Cole TJ, Lobstein T. Extended international (IOTF) body mass index cut-offs for thinness, overweight and obesity. Pediatr Obes. 2012 Aug;7(4):284-94.

20. Farias Júnior JC, Lopes AS, Mota J, Santos MP, Ribeiro JC, Hallal PC. Validity and reproducibility of a physical activity questionnaire for adolescents: adapting the SelfAdministered Physical Activity Checklist. Rev Bras Epidemiol. 2012;15(1):198-210.

21. World Health Organization. Global recommendations on physical activity for health. Geneva, Switzerland; 2010 [Citado em 2018 jan 02]. Disponível em: http://www.who. int/dietphysicalactivity/factsheet_recommendations/en/.
22. Sawka KJ, McCormack GR, Nettel-Aguirre A, Hawe P, Doyle-Baker PK. Friendship networks and physical activity and sedentary behavior among youth: a systematized. Int J Behav Nutr Phys Act. 2013;10(1):130.

23. Mendonça G, Farias Júnior JC. Physical activity and social support in adolescents: analysis of different types and sources of social support. J Sports Sci. 2015;33(18):1942-51.

24. Silva SM, Knuth AG, Duca GFD, Camargo MBJ, Cruz SH, Castagno $\mathrm{V}$, et al. Individual and collective sports practice and associated factors among adolescents belonging to a birth cohort study. Rev Bras Educ Fis Esp. 2009;23(3):263-74.

25. Mendonça G, Cheng LA, Farias Júnior JC. Practice of physical activity patterns in adolescents in a city of Northeastern Brazil. Ciênc Saúde Coletiva. 2018;23(7):2443-51.

26. Bengoechea EG, Sabiston CM, Ahmed R, Farnoush M. Exploring links to unorganized and organized physical activity during adolescence: the role of gender, socioeconomic status, weight status, and enjoyment of physical education. Res QExerc Sport. 2010;81(1):7-16.

Recebido: 09/07/2019

Aprovado: 10/02/2020

\section{Como citar este artigo:}

Mendonça G, Mendes JO, Lucena JMS, Farias Júnior JC. Os fatores sociodemográficos moderam a associação da prática de atividade física dos pais $e$ amigos com o nível de atividade física dos adolescentes? Rev Bras Ativ Fis Saúde. 2019;24:e0092. DOI: 10.12820/rbafs.24e0092 